\title{
Newly proposed insulin resistance indexes called TyG-NC and TyG-NHtR show efficacy in diagnosing the metabolic syndrome
}

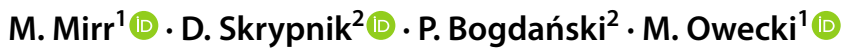

Received: 24 March 2021 / Accepted: 2 June 2021 / Published online: 16 June 2021

(c) The Author(s) 2021

\begin{abstract}
Purpose Obesity and insulin resistance are considered cardinal to the pathophysiology of metabolic syndrome. Several simple indexes of insulin resistance calculated from biochemical or anthropometric variables have been proposed. The study aimed to assess the diagnostic accuracy of indirect insulin resistance indicators in detecting metabolic syndrome in non-diabetic patients, including TG/HDLc, METS-IR, TyG, TyG-BMI, TyG-WC, TyG-WHtR, and new indicators TyG-NC (TyG-neck circumference) and TyG-NHtR (Tyg-neck circumference to height ratio).

Methods The diagnostic accuracy of eight insulin resistance indexes was assessed using the receiver operating characteristic curves (ROC curves) in 665 adult non-diabetic patients. Then, the analysis was performed after the division into groups with proper body mass index, overweight and obese.

Results All indexes achieved significant diagnostic accuracy, with the highest AUC (area under the curve) for TyG (0.888) and $\mathrm{Tg} / \mathrm{HDLc}(0.874)$. The highest diagnostic performance in group with the proper body mass index was shown for TyG (0.909) and TyG-BMI (0.879). The highest accuracy in the group of overweight individuals was presented by TyG $(0.884)$ and TG/HDLc (0.855). TG/HDLc and TyG showed the highest AUC (0.880 and 0.877, respectively) in the group with obesity. Both TyG-NC and TyG-NHtR reached significant areas under the curve, which makes them useful diagnostic tests in metabolic syndrome.

Conclusions Indirect indices of insulin resistance, including proposed TyG-NC and TyG-NHtR, show an essential diagnostic value in diagnosing metabolic syndrome. TyG and TG/HDLc seem to be the most useful in the Caucasian population.
\end{abstract}

Keywords Insulin resistance $\cdot$ Metabolic syndrome $\cdot$ Obesity

\section{Introduction}

Metabolic syndrome is a set of features that increase the risk of developing cardiovascular diseases and diabetes [1]. Obesity and insulin resistance are considered cardinal to the pathophysiology of metabolic syndrome [1,2]. Insulin resistance (IR) is a state of decreased tissue sensitivity to insulin, a glycemic-lowering hormone [3]. Insulin resistance is considered a strong risk factor not only for type 2

M. Mirr

malgorzata.tapolska@gmail.com

1 Department of Public Health, Poznan University of Medical Sciences, Rokietnicka St. 4, 60-806 Poznan, Poland

2 Department of Treatment of Obesity, Metabolic Disorders and Clinical Dietetics, Poznan University of Medical Sciences, Szamarzewskiego St. 82/84, 60-569 Poznan, Poland diabetes but also for cardiovascular complications, including hypertension and non-alcoholic fatty liver disease (NAFLD) [3-5]. The hyperinsulinemic euglycemic clamp (HEC) is the gold standard in assessing the insulin sensitivity of peripheral tissues $[4,6]$. This method consists of continuous insulin infusion until the serum concentration of $100 \mathrm{mIU} / \mathrm{L}$ is achieved and maintained, and simultaneous intravenous glucose infusion $[4,7]$. During exogenous hyperinsulinemia, insulin production by the pancreas and the hepatic glucose production is blocked, and the amount of glucose administered reflects its tissue uptake, and, thus, indirectly insulin sensitivity $[4,8]$. However, because this method is complicated, time- and resource-consuming, insulin resistance is most often assessed using simpler indicators $[4,6]$.

A commonly used indicator that strongly correlates with insulin resistance assessed by HEC is the homeostatic model assessment for insulin resistance (HOMA-IR), calculated based on fasting glucose and insulin levels [7]. This 
method's use is also limited in everyday practice by the relatively high cost of measuring insulin concentration [9]. Several simple indexes of insulin resistance calculated from biochemical or anthropometric variables have been proposed [4, 9]. The triglyceride-glucose (TyG) index derived from circulating triglycerides and glucose concentrations was shown to be comparable or even more predictive than HOMA-IR in assessing the risk of insulin resistance-related conditions [5, 10] TyG strongly correlates with insulin resistance assessed by HEC and shows high sensitivity and specificity in the diagnosis of insulin resistance [11].

The diagnostic value of TyG in the diagnosis of metabolic syndrome has also been shown [12]. In recent years, several indices calculated as products of TyG and anthropometric indices have been proposed, including TyG-waist circumference (TyG-WC), TyG-waist to height ratio (TyGWHtR), and TyG-body mass index (TyG-BMI) [13]. The diagnostic accuracy of these indices in metabolic syndrome has been shown [13]. The relationship of insulin resistance indices with the metabolic syndrome components, especially hypertension and prehypertension state, was investigated [9, 14-16]. In a study by Zeng et al., TyG, TyG-BMI, TyG-WC, and TyG-WHtR presented positive correlations with systolic and diastolic blood pressure in individuals with proper body mass index [16]. In the research by Bala et al., TyG, TyGBMI, and TyG-WC were independently associated with the presence of hypertension [9]. Moreover, Zheng and Mao identified TyG as the predictor of incident hypertension in a follow-up study [17].

A simple insulin resistance indicator TG/HDLc (triglicerides to high-density lipoprotein cholesterol), which is calculated as the ratio between triglycerides and high-density cholesterol concentrations, has also been proposed, and it was shown to be a marker for cardiometabolic and type 2 diabetes risk $[18,19]$. It was also demonstrated that TG/ HDLc is associated with hypertension [9].

Metabolic score for IR (METS-IR) is a novel score to assess insulin resistance defined as $\operatorname{Ln}((2 \times$ fasting glucose $)+$ fasting triglycerides $) \times$ body mass index $) /(\operatorname{Ln}$ (highdensity lipoprotein cholesterol)) [20]. A significant correlation between METS-IR and intravisceral, intrahepatic $(\rho=0.636, P<0.001)$ and intrapancreatic fat was presented [20]. METS-IR also correlated with fasting insulin levels [20]. The ability of METS-IR to predict type 2 diabetes in a 2-year follow-up study was evaluated, and it was presented that the group in the highest quartile of METS-IR had the highest risk to develop diabetes [20]. METS-IR was shown to correlate positively with blood pressure values and is strongly associated with hypertension in normal weight individuals [14]. These findings suggest that METS-IR may also be associated with metabolic syndrome. To date, the usefulness of METS-IR in the diagnosis of metabolic syndrome has not been studied.
Neck circumference (NC) is one of the anthropometric indicators of obesity, but also cardiovascular risk and metabolic syndrome [21, 22]. It seems that since the metabolic syndrome consists of excess body weight, insulin resistance, and hypertension, the combination of $\mathrm{NC}$ and TyG may appear more accurate than either of these indicators alone.

The study aimed to compare the usefulness of indirect insulin resistance indicators in detecting metabolic syndrome in non-diabetic patients, including the derivatives of TyG, and TG/HDLc. We also investigated the usefulness of METS-IR in the diagnosis of metabolic syndrome, which was for the first time to our knowledge. Then, we proposed novel indicators TyG-NC (TyG-neck circumference) and TyG-NHtR (Tyg-neck circumference to height ratio) and tested their usefulness in metabolic syndrome detection before the onset of diabetes. These new indicators are, to our knowledge, our concept and appear in medical literature for the first time.

\section{Materials and methods}

The cross-sectional study included non-diabetic participants aged 18 and over. Individuals with previously diagnosed diabetes and fasting plasma glucose above $125 \mathrm{mg} / \mathrm{dL}$ were excluded from the study to analyze the group of patients before the onset of diabetes. Patients taking lipid-lowering drugs and individuals with severe hypertriglyceridemia (plasma triglycerides $>500 \mathrm{mg} / \mathrm{dL}$ ) were also excluded from the study. The study was approved by the Ethical Committee of the Poznan University of Medical Sciences (approval number 359/15).

Clinical data of each patient was collected, and laboratory tests were performed.

\section{Laboratory tests}

The fasting serum concentrations of glucose (FPG), triglycerides (TG), and high-density lipoprotein cholesterol (HDLc) were assessed.

\section{Anthropometric measurements and calculation of anthropometric indexes}

The anthropometric measurements of each participant were carried out in the morning, fasting (at least $12 \mathrm{~h}$ after last meal):

- measurement of body height in an upright standing position without shoes with an accuracy of $0.5 \mathrm{~cm}$,

- measurement of waist circumference (WC) at the approximate midpoint between the lower margin of the last pal- 
Table 1 Group characteristics

\begin{tabular}{lcc}
\hline Feature & Mean \pm SD & Median \\
\hline Age (years) & $53.9 \pm 14.5$ & 57.0 \\
Body mass $(\mathrm{kg})$ & $75.4 \pm 15.2$ & 73.5 \\
Height $(\mathrm{cm})$ & $165.8 \pm 9.1$ & 165.0 \\
Waist circumference $(\mathrm{cm})$ & $92.9 \pm 14.2$ & 92.0 \\
Neck circumference $(\mathrm{cm})$ & $36.2 \pm 3.7$ & 36.0 \\
BMI $\left(\mathrm{kg} / \mathrm{m}^{2}\right)$ & $27.4 \pm 4.8$ & 26.9 \\
WHtR & $0.56 \pm 0.08$ & 0.56 \\
NHtR & $0.22 \pm 0.02$ & 0.22 \\
FPG $(\mathrm{mg} / \mathrm{dL})$ & $90.4 \pm 11.1$ & 89.0 \\
TC (mg/dL) & $203.6 \pm 42.0$ & 201.0 \\
HDLc (mg/dL) & $65.9 \pm 17.2$ & 63.0 \\
LDLc (mg/dL) & $111.1 \pm 39.7$ & 106.0 \\
TG (mg/dL) & $142.2 \pm 78.2$ & 125.0 \\
Systolic BP (mmHg) & $133.6 \pm 19.3$ & 132.0 \\
Diastolic BP(mmHg) & $80.9 \pm 11.1$ & 80.0 \\
TyG & $8.6 \pm 0.6$ & 8.6 \\
TyG-BMI & $236.7 \pm 48.4$ & 231.6 \\
TyG-WC & $803.9 \pm 146.8$ & 802.3 \\
TyG-WHtR & $4.9 \pm 0.9$ & 4.8 \\
TyG-NC & $313.0 \pm 42.3$ & 309.5 \\
TyG-NHtR & $1.9 \pm 0.2$ & 1.9 \\
METS-IR & $38.2 \pm 8.6$ & 37.3 \\
TG/HDLc & $2.4 \pm 1.7$ & 1.96 \\
\hline
\end{tabular}

pable rib and the top of the iliac crest, using an unstrechable tape(in units of $\mathrm{cm}$ ),

- measurement of neck circumference (NC) in the midway of the neck, between midcervical spine and midanterior neck, to within $0.5 \mathrm{~cm}$, with plastic unstrechable tape

- measurement of body mass without shoes in underwear assessed using the certified electronic weighing scale with the accuracy of $0.1 \mathrm{~kg}$ (Radwag, Poland).

- body mass index (BMI), defined as the body mass divided by the square of the body height (expressed in units of $\mathrm{kg} / \mathrm{m}^{2}$ ),

- waist to height ratio (WHtR), defined as the waist circumference divided by the body height

- neck to height ratio (NHtR) defined as the neck circumference divided by the body height

\section{Blood pressure measurements}

The arterial blood pressure measurements were performed using Digital electronic tensiometer (Omron Corporation ${ }^{\mathrm{TM}}$, Kyoto, Japan), following the European Society of Hypertension and European Society of Cardiology (ESH/ESC) recommendations from 2013 [23]. Measurement was carried out twice, and if the values were significantly different, the measure was averaged out. Both systolic and diastolic blood pressure values were measured with an accuracy of $2 \mathrm{mmHg}$.

\section{Insulin resistance indexes}

Insulin resistance indicators were calculated according to the following formulas:

$$
\text { TyG }=\text { Ln [fasting TG }(\mathrm{mg} / \mathrm{dL}) \times \mathrm{FPG}(\mathrm{mg} / \mathrm{dL}) / 2],[14]
$$$$
\text { TyG-BMI }=\text { TyG } \times \text { BMI, [9] }
$$

TyG-WC $=$ TyG $\times$ WC, [9]

TyG-WHtR $=$ TyG $\times$ WHtR, [13]

TyG-NC $=$ TyG $\times$ NC,

TyG-NHtR $=$ TyG $\times$ NHtR,

TG/HDLc $=$ fasting TG $(\mathrm{mg} / \mathrm{dL}) /$ fasting HDL cholesterol (mg/dL), [14]

METS-IR $=\operatorname{Ln}[(2 \times$ FPG $(\mathrm{mg} / \mathrm{dL})+$ fasting TG $(\mathrm{mg} /$ $\left.\mathrm{dL})] \times \mathrm{BMI}\left(\mathrm{kg} / \mathrm{m}^{2}\right)\right) /(\mathrm{Ln}[\mathrm{HDLc}(\mathrm{mg} / \mathrm{dL}])$. [20]

\section{Metabolic syndrome criteria}

Metabolic syndrome criteria were defined as the presence of any 3 of 5 risk factors established by the International Diabetes Federation Task Force on Epidemiology and Prevention in 2009 for the Caucasians [24]:

- triglycerides: $\geq 150 \mathrm{mg} / \mathrm{dL}$ or specific treatment for this lipid abnormality

- HDL cholesterol: $<40 \mathrm{mg} / \mathrm{dL}$ in males, $<50 \mathrm{mg} / \mathrm{dL}$ in females, or specific treatment for this lipid abnormality

- blood pressure (BP): systolic BP $>130$ or diastolic $\mathrm{BP}>85 \mathrm{~mm} \mathrm{Hg}$, or treatment of previously diagnosed hypertension

- fasting plasma glucose (FPG): $\geq 100 \mathrm{mg} / \mathrm{dL}$ or previously diagnosed type 2 diabetes (the individuals diagnosed with diabetes were excluded in this study)

- waist circumference $\geq 80 \mathrm{~cm}$ in females and $\geq 94 \mathrm{~cm}$ in males [24]

\section{Statistical analysis}

Statistical analysis was performed with the Statistica v13 and Microsoft Excel Analyze it software. The predictive accuracy of insulin resistance indexes was assessed using the receiver operating characteristic curves (ROC curves). For each of the insulin resistance indexes, the calculations of the area under the ROC curve (AUC-ROC) were made. If the AUC was significantly greater than 0.5 , the calculations of sensitivity and specificity were carried out. The optimal thresholds for each index were selected, choosing the highest Youden's index value. First, a non-grouping analysis was performed, then curves were plotted, and calculations 


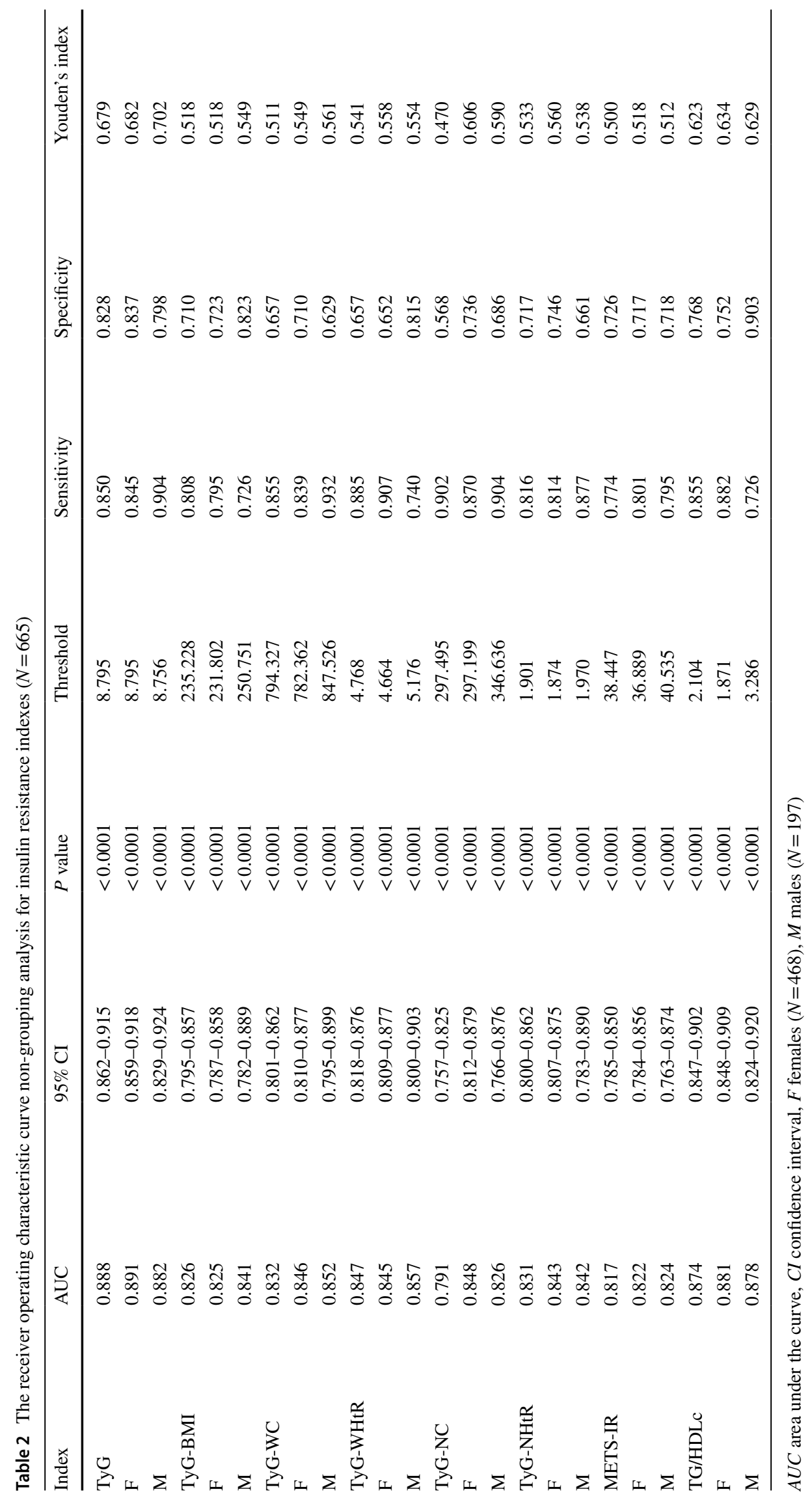




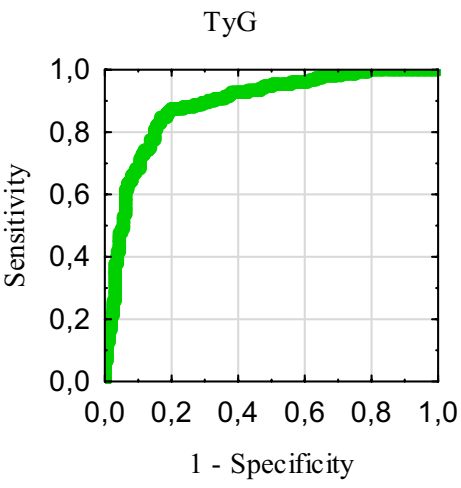

TyG-WHtR

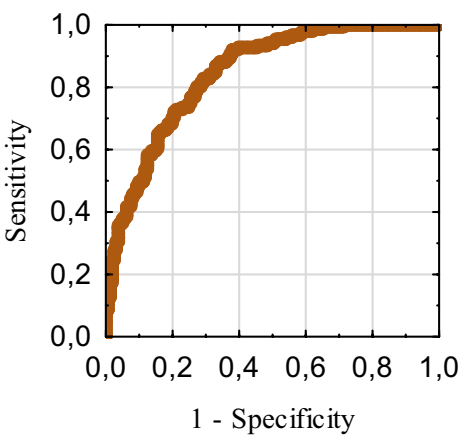

METS-IR

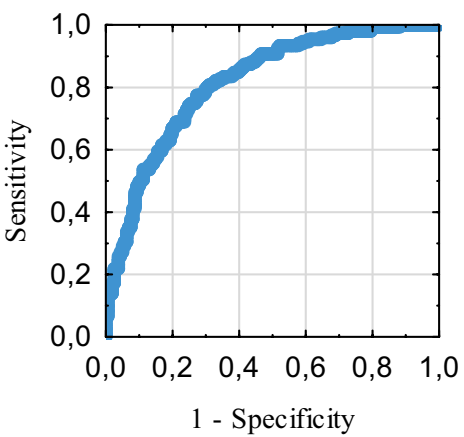

TyG-BMI

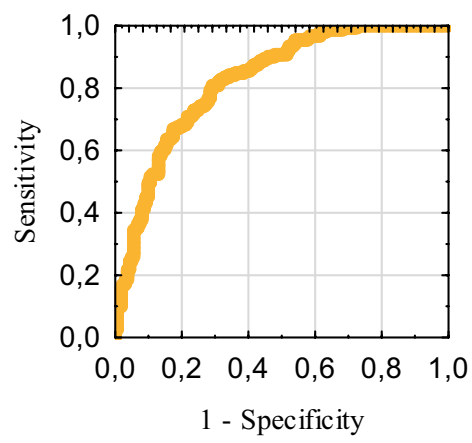

TyG-NC

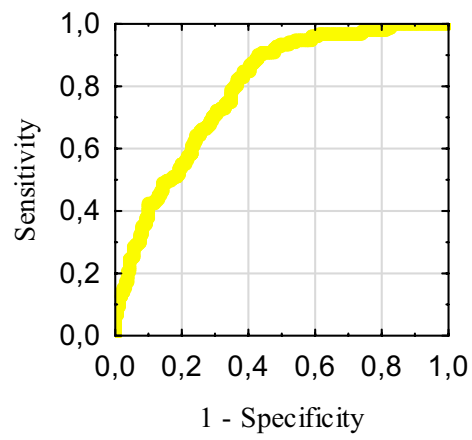

TG/HDLc

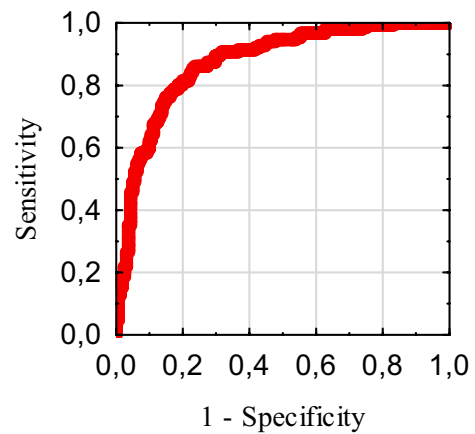

TyG-WC

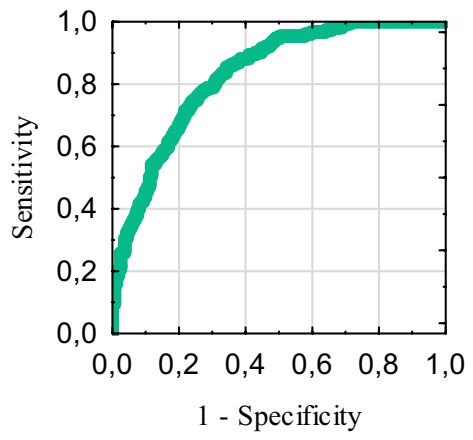

TyG-NHtR

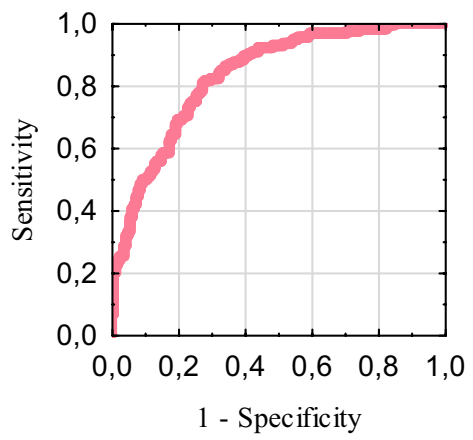

Comparison

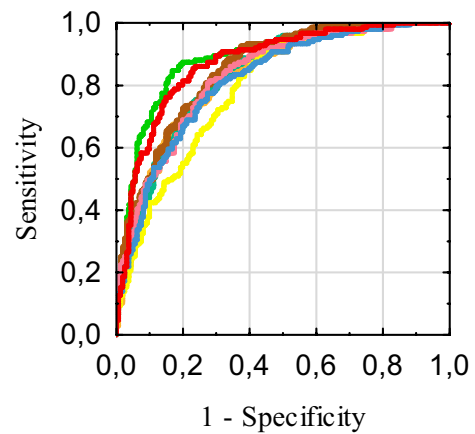

Fig. 1 Receiver operating characteristic curves non-grouping analysis

were made for normal BMI group (BMI between 18.5 and $24.99 \mathrm{~kg} / \mathrm{m}^{2}$ ), overweight (BMI between 25 and $29.99 \mathrm{~kg} /$ $\mathrm{m}^{2}$ ) and obese group (BMI $\geq 30 \mathrm{~kg} / \mathrm{m}^{2}$ ), respectively. The curves were plotted also separately for males and females to establish separate thresholds.

The minimal sample size was estimated using MedCalc software for the area under the ROC curve test. Based on previous reports, we assumed the predicted AUC as 0.75, type I error as 0.05 , type II error as $0.20[12,13]$. We estimated the ratio of negative to positive sample sizes as 0.6. The required sample size was estimated as 40 , which we considered the minimum number of patients in each analyzed subgroup by gender and BMI.

\section{Results}

The study included 665 individuals. Females constituted $70.4 \%$ of the group. Obesity was diagnosed in $26.5 \%$ of the group. Impaired fasting plasma glucose was present in $19.4 \%$ of the study group. $31.4 \%$ of the examined group were previously diagnosed with hypertension. $35.2 \%$ met the criteria of the metabolic syndrome. The values of the anthropometric, laboratory, and insulin resistance indices are presented in the Table 1.

The results of ROC-AUC analysis, 95\% confidence interval, optimal thresholds, corresponding sensitivity, specificity, and Youden's index values for each insulin resistance index for the whole study group are presented in the Table 2 . 


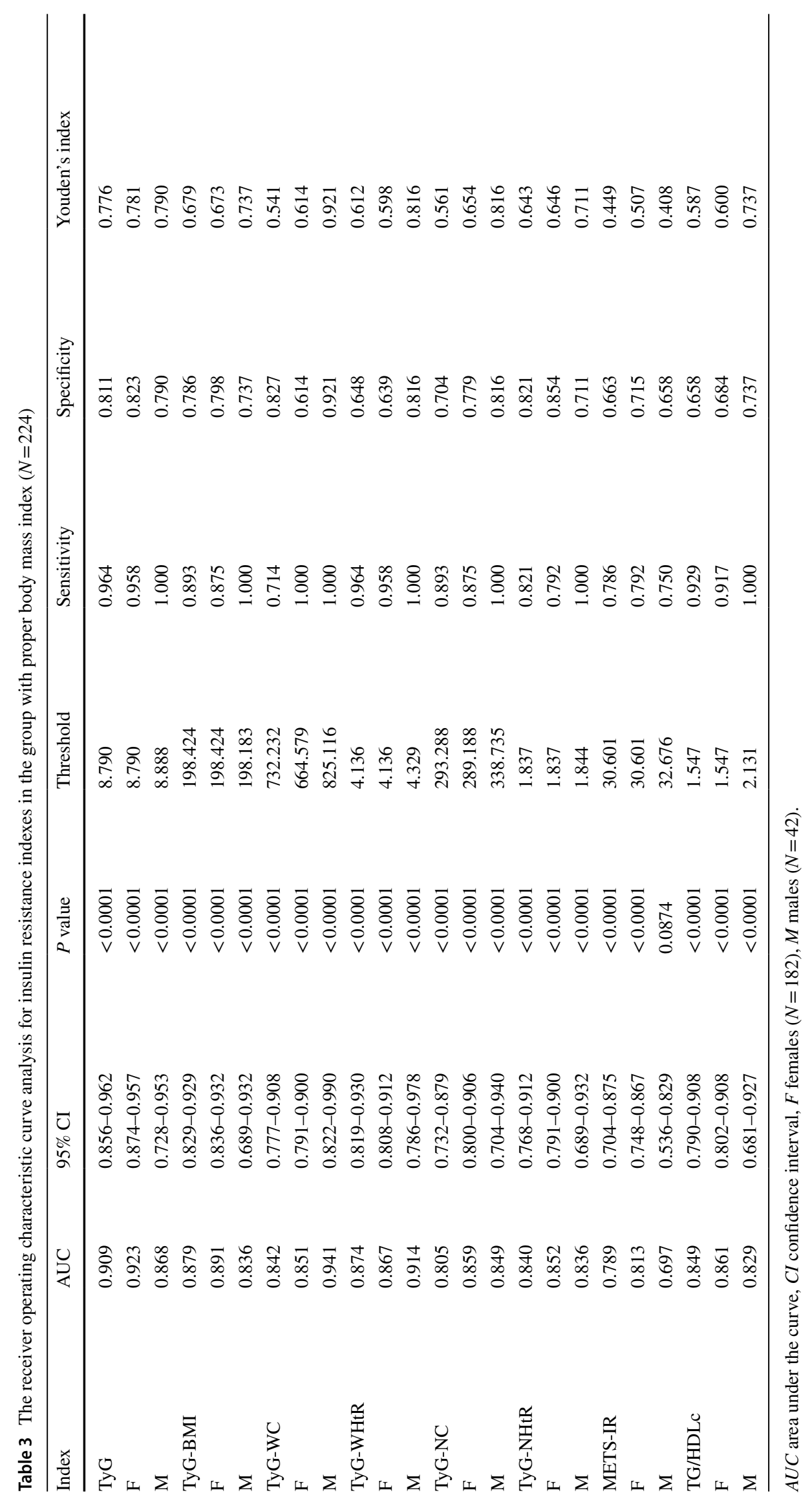


TyG

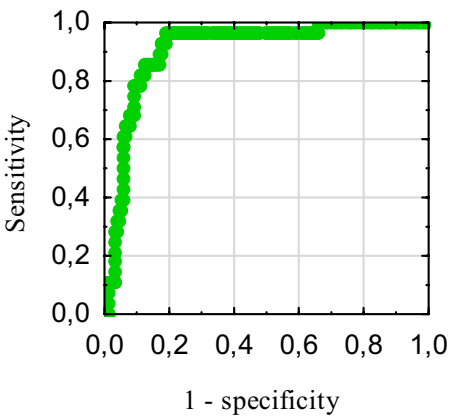

TyG-WHtR

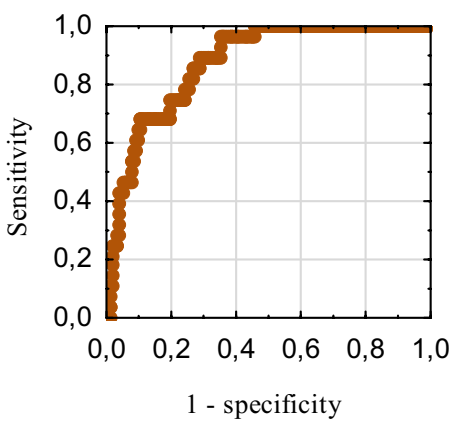

METS-IR

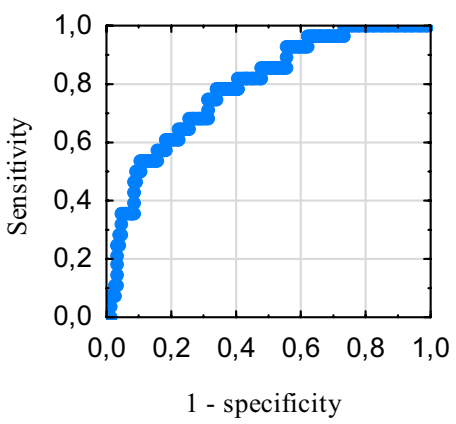

TyG-BMI

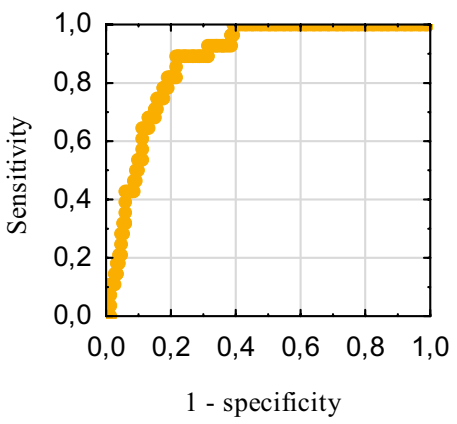

TyG-NC

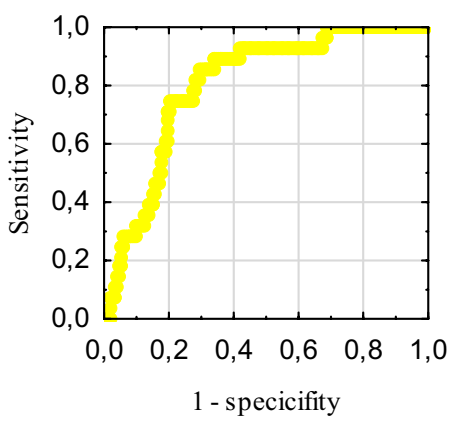

TG/HDLc

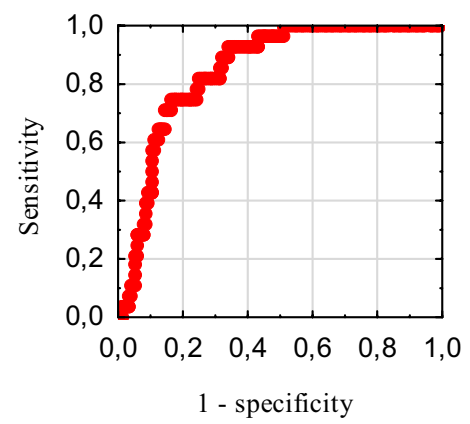

TyG-WC

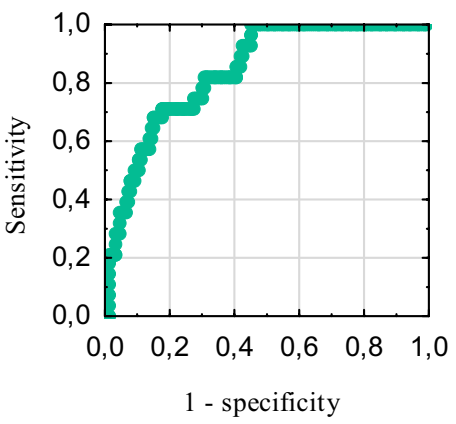

TyG-NHtR

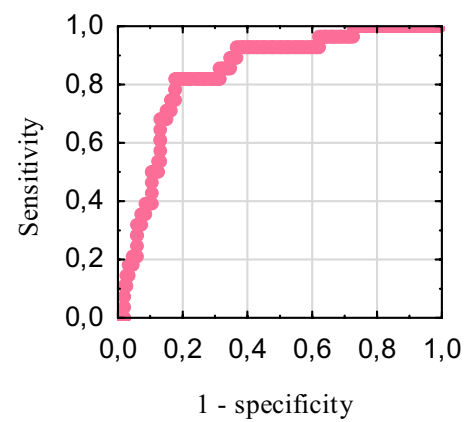

Comparison

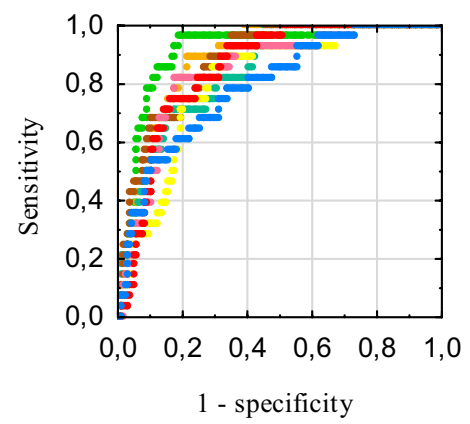

Fig. 2 Receiver operating characteristic curves in the group with proper body mass index

The receiver operating curves for the tested indices and the comparison of them are presented in the Fig. 1. Testing of ROC-AUC showed that all analyzed insulin resistance indexes may discriminate the metabolic syndrome from healthy individuals. The analysis presented the highest area under the curve for TyG and TG/HDLc and the lowest area under the curve for TyG-NC.

The results of the ROC-AUC, 95\% confidence intervals, optimal thresholds, corresponding sensitivity, specificity, and Youden's index values for insulin resistance indexes in the group with proper body mass index are presented in the Table 3. All indexes achieved significant diagnostic accuracy, with the highest AUC for TyG and the lowest for METS-IR. Only the AUC for METS-IR in males did not reach statistical significance. The receiver operating curves for this group are shown in the Fig. 2.

The results of the ROC curve analysis for the overweight group are shown in the Table 4. As in the previous group, all indicators showed a significant AUC. The highest AUC was achieved by TyG and TG/HDLc, and the lowest by TyGNC. The receiver operating curves for this group are demonstrated in the Fig. 3.

The results of the ROC curve testing in the group with obesity are presented in the Table 5. All indexes achieved significant diagnostic accuracy, with the highest AUC for TG/HDLc and TyG, and the lowest for TyG-NC. The receiver operating curves for this group are showed in the Fig. 4. 


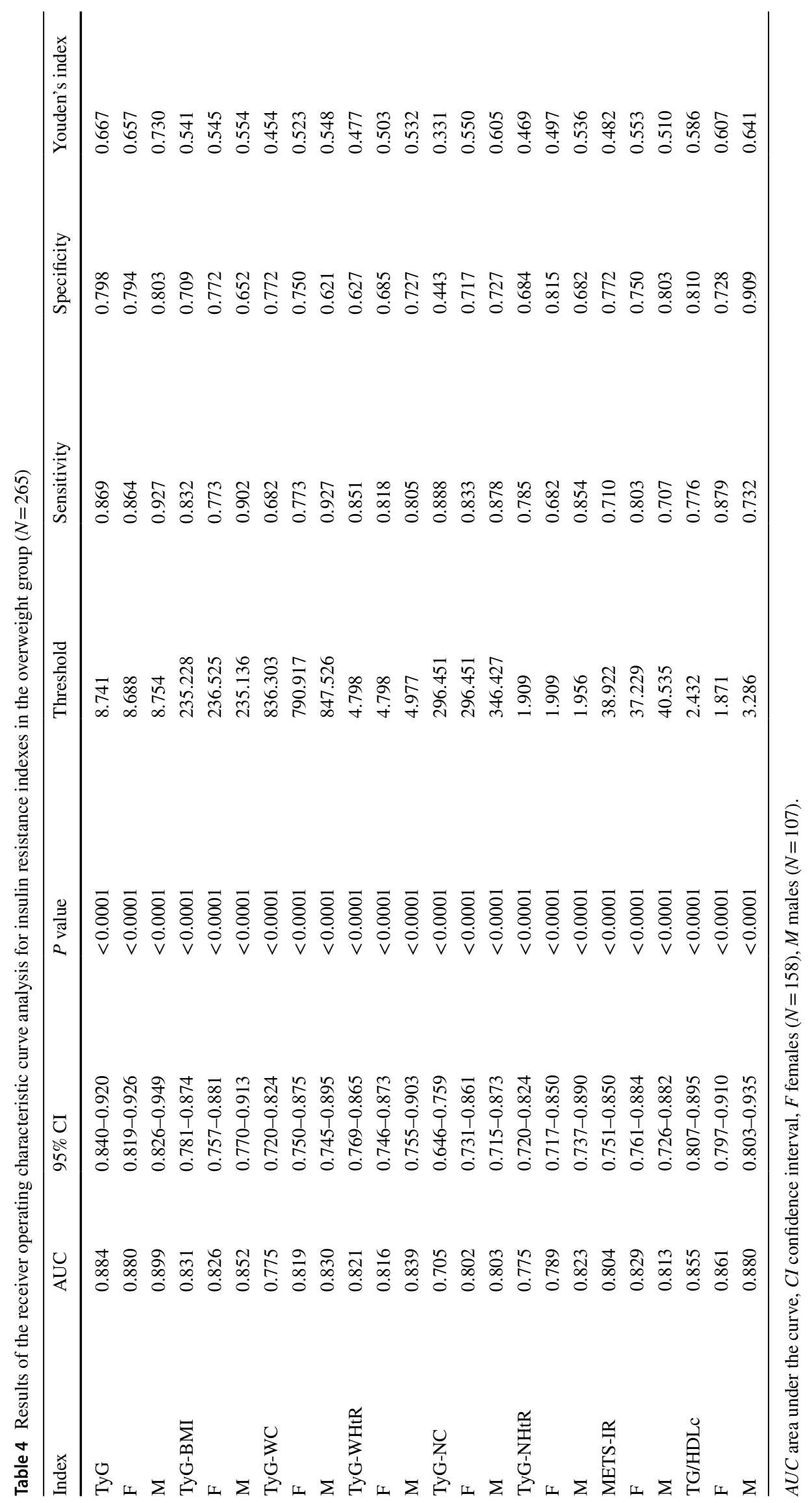


TyG

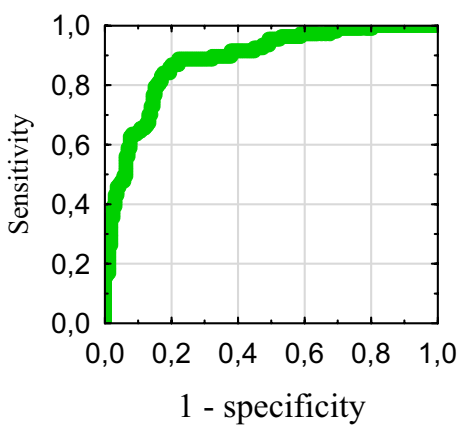

TyG-WHtR

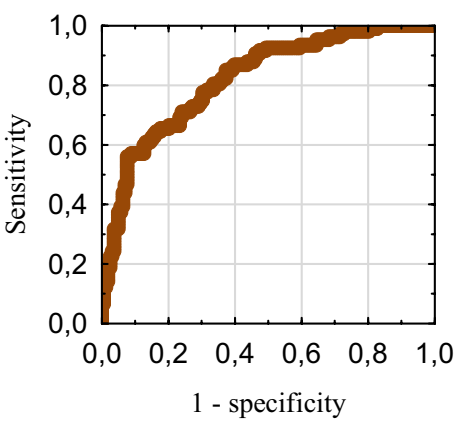

METS-IR

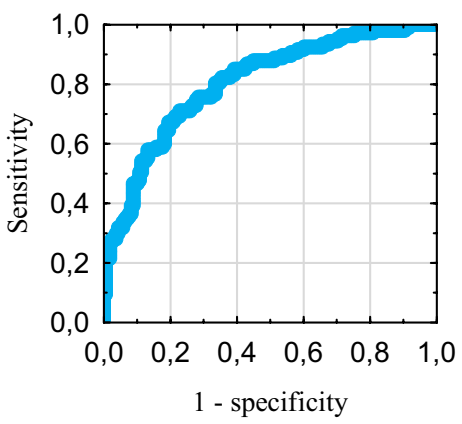

TyG-BMI

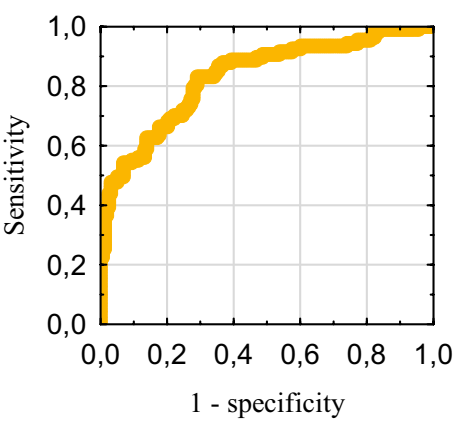

TyG-NC

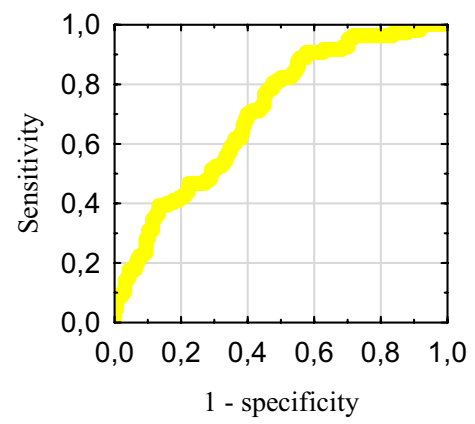

TG/HDLc

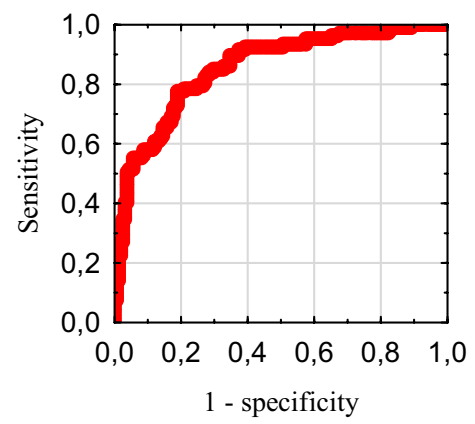

TyG-WC

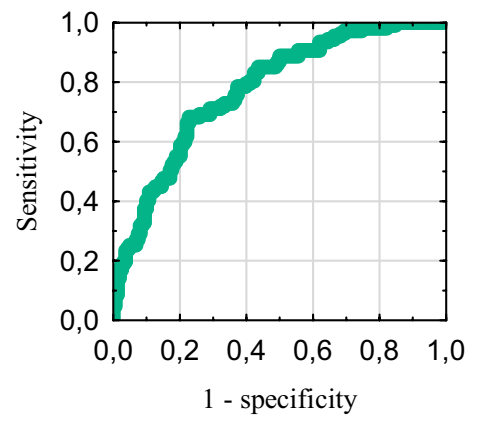

TyG-NHtR

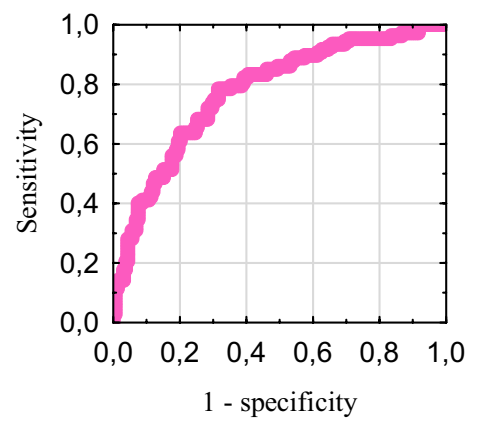

Comparison

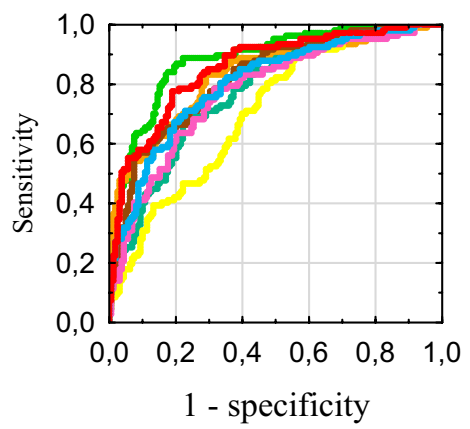

Fig. 3 Receiver operating characteristic curves in the overweight group

\section{Discussion}

Our study confirms previous reports that insulin resistance's indirect indices may be of diagnostic value in diagnosing metabolic syndrome $[12,13,25]$. Interestingly, it was demonstrated that TyG has a better capacity in predicting metabolic syndrome than HOMA-IR, which emphasizes the role of indirect indices of insulin resistance in daily practice [10, 12]. Several studies have compared the usefulness of insulin resistance indicators $[13,25,26]$. Yu et al. compared TyG, METS-IR, and TG/HDLc in diagnosing metabolic syndrome, identifying TyG as the one with the highest diagnostic accuracy, followed by TG/HDLc and METS-IR, which is consistent with our results [26]. A study comparing
IR indices in metabolic syndrome by Raimi et al. showed the largest AUC for metabolic syndrome detection for TyGWHtR, followed by TyG-WC, TyG-BMI, and eventually, TyG index, which is inconsistent with our study [13]. However, the discrepancy in the markers' usefulness across countries has already been suggested and it is possible that ethnic differentiation can explain these differences [13]. A study by Lee et al. suggests TyG as an indicator of metabolically obese but normal weight, which is consistent with our observations that $\mathrm{TyG}$ is the best indicator for recognizing the metabolic syndrome features in a group of patients with normal body weight [5]. In the study by Lim et al., TyG-BMI was found to predict insulin resistance better than TyG, TyGWC, and TyG-WHtR [25]. In this research, TyG and obesity indices' combinations showed better insulin resistance 
Table 5 Results of the receiver operating characteristic curve analysis for insulin resistance indexes in the obese group $(N=176)$

\begin{tabular}{|c|c|c|c|c|c|c|c|}
\hline Index & AUC & $95 \%$ CI & $P$ value & Threshold & Sensitivity & Specificity & Youden's index \\
\hline TyG & 0.877 & $0.819-0.922$ & $<0.0001$ & 8.765 & 0.849 & 0.870 & 0.719 \\
\hline $\mathrm{F}$ & 0.870 & $0.799-0.923$ & $<0.0001$ & 8.813 & 0.817 & 0.895 & 0.712 \\
\hline M & 0.887 & $0.763-0.960$ & $<0.0001$ & 8.719 & 0.857 & 0.900 & 0.757 \\
\hline TyG-BMI & 0.798 & $0.698-0.828$ & $<0.0001$ & 287.928 & 0.778 & 0.675 & 0.453 \\
\hline $\mathrm{F}$ & 0.749 & $0.665-0.822$ & $<0.0001$ & 275.782 & 0.887 & 0.544 & 0.431 \\
\hline M & 0.814 & $0.676-0.912$ & $<0.0001$ & 287.116 & 0.786 & 0.750 & 0.536 \\
\hline TyG-WC & 0.767 & $0.697-0.827$ & $<0.0001$ & 951.726 & 0.657 & 0.753 & 0.410 \\
\hline $\mathrm{F}$ & 0.779 & $0.697-0.847$ & $<0.0001$ & 920.556 & 0.704 & 0.755 & 0.459 \\
\hline M & 0.825 & $0.688-0.919$ & $<0.0001$ & 987.361 & 0.857 & 0.800 & 0.657 \\
\hline TyG-WHtR & 0.789 & $0.721-0.846$ & $<0.0001$ & 5.745 & 0.727 & 0.792 & 0.520 \\
\hline $\mathrm{F}$ & 0.769 & $0.686-0.839$ & $<0.0001$ & 5.668 & 0.747 & 0.754 & 0.501 \\
\hline M & 0.850 & $0.717-0.937$ & $<0.0001$ & 5.745 & 0.821 & 0.800 & 0.621 \\
\hline TyG-NC & 0.732 & $0.660-0.796$ & $<0.0001$ & 339.246 & 0.616 & 0.766 & 0.382 \\
\hline $\mathrm{F}$ & 0.786 & $0.704-0.853$ & $<0.0001$ & 333.076 & 0.549 & 0.877 & 0.426 \\
\hline M & 0.775 & $0.631-0.883$ & $<0.0001$ & 363.852 & 0.786 & 0.650 & 0.436 \\
\hline TyG-NHtR & 0.779 & $0.710-0.838$ & $<0.0001$ & 2.081 & 0.636 & 0.792 & 0.429 \\
\hline $\mathrm{F}$ & 0.785 & $0.703-0.852$ & $<0.0001$ & 2.045 & 0.648 & 0.772 & 0.420 \\
\hline M & 0.816 & $0.678-0.913$ & $<0.0001$ & 2.206 & 0.607 & 0.950 & 0.557 \\
\hline METS-IR & 0.762 & $0.692-0.823$ & $<0.0001$ & 44.425 & 0.899 & 0.520 & 0.419 \\
\hline $\mathrm{F}$ & 0.747 & $0.662-0.819$ & $<0.0001$ & 43.215 & 0.958 & 0.491 & 0.449 \\
\hline M & 0.811 & $0.672-0.909$ & $<0.0001$ & 47.961 & 0.750 & 0.800 & 0.550 \\
\hline TG/HDLc & 0.880 & $0.823-0.924$ & $<0.0001$ & 2.720 & 0.768 & 0.909 & 0.677 \\
\hline $\mathrm{F}$ & 0.877 & $0.807-0.929$ & $<0.0001$ & 2.489 & 0.775 & 0.930 & 0.705 \\
\hline M & 0.902 & $0.781-0.969$ & $<0.0001$ & 3.152 & 0.821 & 0.950 & 0.771 \\
\hline
\end{tabular}

$A U C A$ indicate area under the curve, $C I$ confidence interval, $F$ females $(N=128), M$ males $(N=48)$ prediction performance than TyG alone [25]. However, our study shows that in the diagnosis of metabolic syndrome, TyG has a better diagnostic value than its products with anthropometric indices. The studied group's characteristics may also be of some importance, as the mean BMI in the study mentioned above was lower than in ours, amounting $23.8 \pm 3.1$, which may partly explain the differences [25].

Neck circumference was suggested as a new promising indicator of metabolic syndrome and cardiovascular risk $[21,22,27]$. ROC curve analysis performed by Laohabut et al. showed that neck circumference may be a useful tool for metabolic syndrome prediction [22]. Yang et al. presented that larger neck circumference is associated with an increased risk of coronary heart disease [27]. Moreover, the neck circumference and waist circumference ability to predict cardiovascular risk and identify the presence of metabolic syndrome seems similar [28, 29]. Since NC and TyG appear to be good indicators of insulin resistance and metabolic syndrome, TyG-NC and TyG-NHtR should potentially be a good representation of the metabolic syndrome features. As expected, both TyG-NC and TyG-NHtR reached the areas under the curve, which makes them useful diagnostic tests. TyG-NHtR seems to have a higher diagnostic value than TyG-NC, however, the application of these two indicators in practice requires further observations.

TG/HDLc, despite its simplicity, seems to be just as useful, if not better, than some of the proposed indicators based on combined biochemical and anthropometric measurements. In our study, TG/HDLc achieved the second-largest ROC-AUC after TyG with high sensitivity and specificity in the whole study group, and the highest diagnostic accuracy in the group of obese individuals.

The study also has some limitations that need to be identified. First, the study was conducted on the Caucasian population, so one should carefully conclude on the usefulness of the investigated indexes in other populations. Moreover, to create a homogenous group of patients and to identify indicators useful in the early diagnosis of metabolic syndrome, patients taking medications for hyperlipidemia, impaired glucose tolerance and diabetes were excluded from the analysis. Therefore, the proposed indicators should be considered early markers of metabolic syndrome that are useful in patients prior to treatment. Separate studies are required to identify the indices useful in the diagnosis of metabolic syndrome in groups of patients during the treatment of impaired glucose tolerance, diabetes, or hyperlipidemia. Furthermore, the cut-off points presented may have 


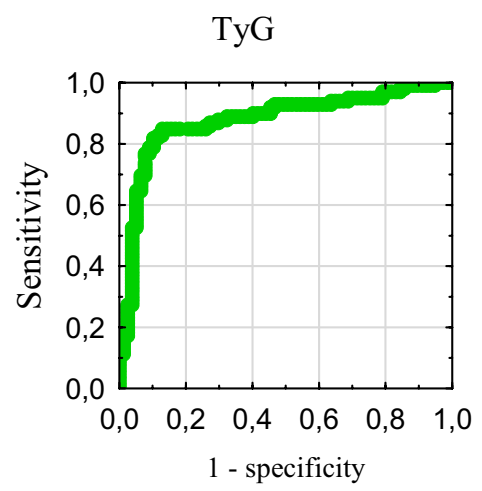

TyG-WHtR

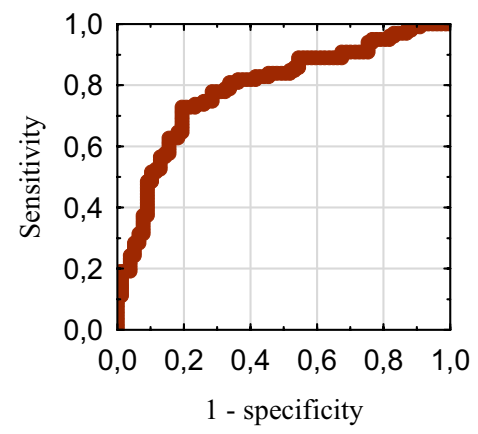

METS-IR

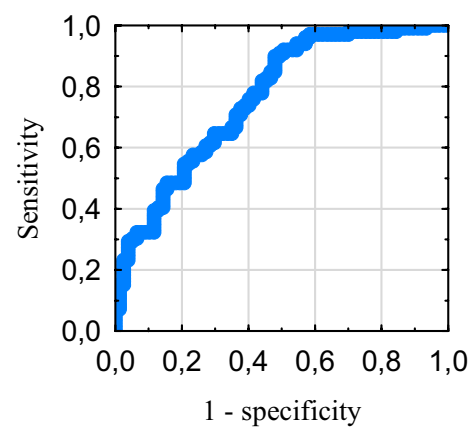

TyG-BMI

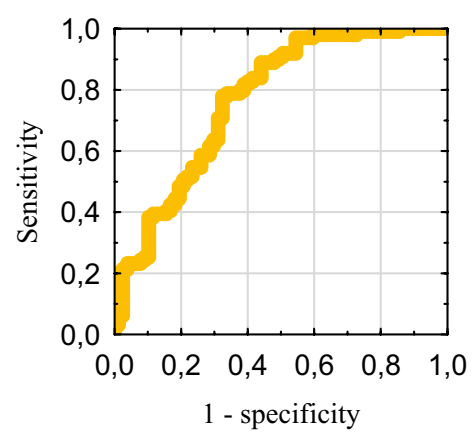

TyG-NC

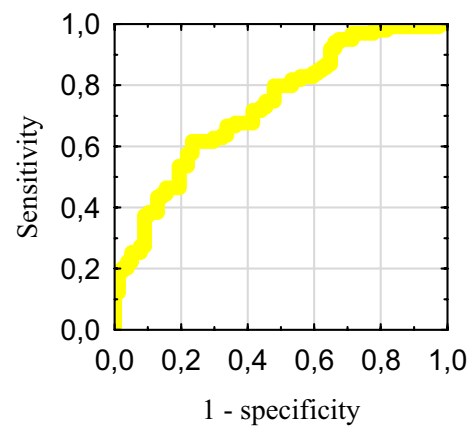

TG/HDLc

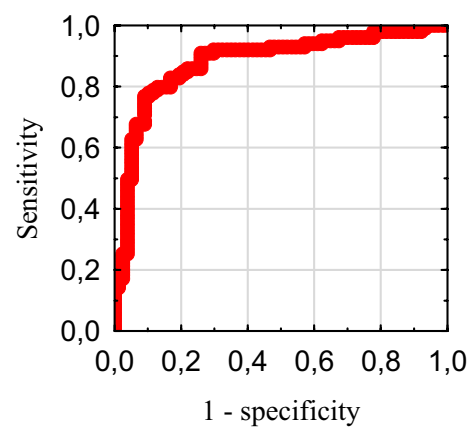

TyG-WC

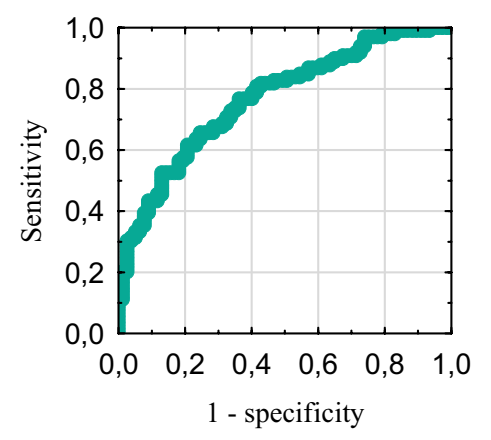

TyG-NHtR

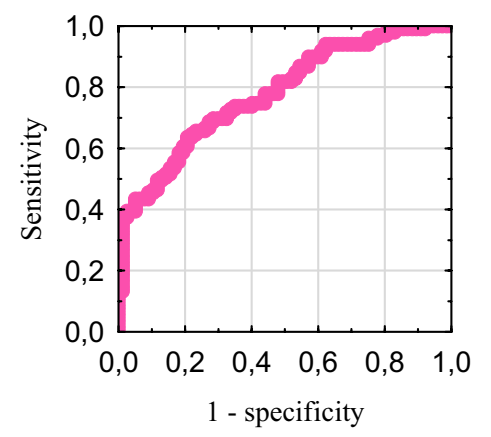

Comparison

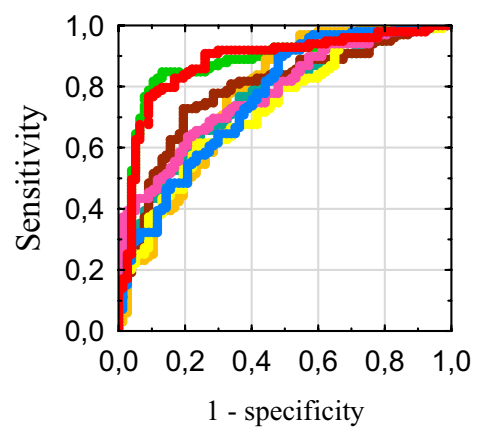

Fig. 4 Receiver operating characteristic curves in the obese group

been affected by unequal gender representation, and gender-specific thresholds should be considered more reliable. Finally, the reported cut-off points should be considered tentative due to the study group's insufficient size to consider the results as reference values for the entire population.

\section{Conclusions}

Indirect indices of insulin resistance, such as TG/HDLc, METS-IR, TyG, and its derivatives, including newly proposed TyG-NC and TyG-NHtR, show an essential diagnostic value in the diagnosis of the metabolic syndrome.
TyG and TG/HDLc seem to be the most useful in the Caucasian population, but further research is needed to clarify the use of individual indicators. TG/HDLc deserves additional emphasis, as calculated from just two biochemical parameters is not inferior to other indicators' diagnostic value, and its use in everyday medical practice should be considered.

Author contributions Conceptualization: MM; Methodology: MM, DS; Resources: DS; Formal analysis: MM; Writing_original draft preparation: MM; Writing-review and editing: MO; Investigation: DS, PB; Project administration: PB; Supervision: MO.

Funding No funding was received for conducting this study. 
Availability of data and material (data transparency) The datasets generated during and/or analyzed during the current study are available from the corresponding author on reasonable request.

Code availability (software application or custom code) Not applicable.

\section{Declarations}

Conflict of interest The authors have no conflicts of interest to declare that are relevant to the content of this article.

Ethics approval The study was approved by the Ethical Committee of the Poznan University of Medical Sciences (approval number 359/15). The study was performed in accordance with the ethical standards as laid down in the 1964 Declaration of Helsinki.

Consent to participate Informed consent was obtained from all individual participants included in the study.

Consent for publication Not applicable.

Open Access This article is licensed under a Creative Commons Attribution 4.0 International License, which permits use, sharing, adaptation, distribution and reproduction in any medium or format, as long as you give appropriate credit to the original author(s) and the source, provide a link to the Creative Commons licence, and indicate if changes were made. The images or other third party material in this article are included in the article's Creative Commons licence, unless indicated otherwise in a credit line to the material. If material is not included in the article's Creative Commons licence and your intended use is not permitted by statutory regulation or exceeds the permitted use, you will need to obtain permission directly from the copyright holder. To view a copy of this licence, visit http://creativecommons.org/licenses/by/4.0/.

\section{References}

1. Samson SL, Garber AJ (2014) Metabolic syndrome. Endocrinol Metab Clin North Am 43(1):1-23. https://doi.org/10.1016/j.ecl. 2013.09.009

2. Eckel RH, Grundy SM, Zimmet PZ (2005) The metabolic syndrome. Lancet Lond Engl 365(9468):1415-1428. https://doi.org/ 10.1210/er.2008-0024

3. De Souza AL, Batista GA, Alegre SM (2017) Assessment of insulin sensitivity by the hyperinsulinemic euglycemic clamp: comparison with the spectral analysis of photoplethysmography. J Diabetes Complicat 31(1):128-133. https://doi.org/10.1016/j. jdiacomp.2016.10.018

4. Spoto B, Pisano A, Zoccali C (2016) Insulin resistance in chronic kidney disease: a systematic review. Am J Physiol Renal Physiol 311(6):F1087-F1108. https://doi.org/10.1152/ajprenal.00340. 2016

5. Lee SB, Kim MK, Kang S, Park K, Kim JH, Baik SJ et al (2019) Triglyceride glucose index is superior to the homeostasis model assessment of insulin resistance for predicting nonalcoholic fatty liver disease in Korean adults. Endocrinol Metab Seoul Korea 34(2):179-186. https://doi.org/10.3803/EnM.2019.34.2.179

6. Łojko D, Owecki M, Suwalska A (2019) Impaired glucose metabolism in bipolar patients: the role of psychiatrists in its detection and management. Int J Environ Res Public Health 16(7):1132. https://doi.org/10.3390/ijerph16071132

7. So A, Sakaguchi K, Okada Y, Morita Y, Yamada T, Miura H et al (2020) Relation between HOMA-IR and insulin sensitivity index determined by hyperinsulinemic-euglycemic clamp analysis during treatment with a sodium-glucose cotransporter 2 inhibitor. Endocr J 67(5):501-507. https://doi.org/10.1507/endocrj. EJ19-0445

8. Kowalchuk C, Teo C, Wilson V, Chintoh A, Lam L, Agarwal SM et al (2017) In male rats, the ability of central insulin to suppress glucose production is impaired by olanzapine, whereas glucose uptake is left intact. J Psychiatry Neurosci JPN 42(6):424-431. https://doi.org/10.1503/jpn.170092

9. Bala C, Gheorghe-Fronea O, Pop D, Pop C, Caloian B, Comsa H et al (2019) The association between six surrogate insulin resistance indexes and hypertension: a population-based study. Metab Syndr Relat Disord 17(6):328-333. https://doi.org/10.1089/met. 2018.0122

10. Vasques ACJ, Novaes FS, de Oliveira M da S, Souza JRM, Yamanaka A, Pareja JC et al (2011) TyG index performs better than HOMA in a Brazilian population: a hyperglycemic clamp validated study. Diabetes Res Clin Pract 93(3):e98-100. https:// doi.org/10.1016/j.diabres.2011.05.030

11. Guerrero-Romero F, Simental-Mendía LE, González-Ortiz M, Martínez-Abundis E, Ramos-Zavala MG, Hernández-González SO et al (2010) The product of triglycerides and glucose, a simple measure of insulin sensitivity. Comparison with the euglycemichyperinsulinemic clamp. J Clin Endocrinol Metab 95(7):33473351. https://doi.org/10.1210/jc.2010-0288

12. Khan SH, Sobia F, Niazi NK, Manzoor SM, Fazal N, Ahmad F (2018) Metabolic clustering of risk factors: evaluation of triglyceride-glucose index (TyG index) for evaluation of insulin resistance. Diabetol Metab Syndr 95(7):3347-3351. https://doi.org/10. 1210/jc.2010-0288

13. Raimi TH, Dele-Ojo BF, Dada SA, Fadare JO, Ajayi DD, Ajayi EA et al (2021) Triglyceride-glucose index and related parameters predicted metabolic syndrome in Nigerians. Metab Syndr Relat Disord 19(2):76-82. https://doi.org/10.1089/met.2020. 0092

14. Liu XZ, Fan J, Pan SJ (2019) METS-IR, a novel simple insulin resistance indexes, is associated with hypertension in normalweight Chinese adults. J Clin Hypertens Greenwich Conn 21(8):1075-1081. https://doi.org/10.1111/jch.13591

15. Fan J, Gao ST, Wang LJ, Qian ZL, Zhou ZQ, Liu XZ (2019) Association of three simple insulin resistance indexes with prehypertension in normoglycemic subjects. Metab Syndr Relat Disord 17(7):374-379. https://doi.org/10.1089/met.2019.0029

16. Zeng ZY, Liu SX, Xu H, Xu X, Liu XZ, Zhao XX (2020) Association of triglyceride glucose index and its combination of obesity indices with prehypertension in lean individuals: a crosssectional study of Chinese adults. J Clin Hypertens Greenwich Conn 22(6):1025-1032. https://doi.org/10.1111/jch.13878

17. Zheng R, Mao Y (2017) Triglyceride and glucose (TyG) index as a predictor of incident hypertension: a 9-year longitudinal population-based study. Lipids Health Dis 16(1):175. https://doi.org/ 10.1186/s12944-017-0562-y

18. Zhang Y, Qin P, Lou Y, Zhao P, Li X, Qie R et al (2020) Association of TG/HDLC ratio trajectory and risk of type 2 diabetes: a retrospective cohort study in China. J Diabetes. https://doi.org/10. 1111/1753-0407.13123 (Online ahead of print)

19. Aguirre M, Briceño Y, Gómez-Pérez R, Zerpa Y, Camacho N, Paoli M (2018) Triglycerides/high density lipoprotein cholesterol ratio as a cardiometabolic risk marker in children and adolescents from Mérida city. Venezuela Endocrinol Diabetes Nutr 65(2):7483. https://doi.org/10.1016/j.endinu.2017.10.010

20. Bello-Chavolla OY, Almeda-Valdes P, Gomez-Velasco D, ViverosRuiz T, Cruz-Bautista I, Romo-Romo A et al (2018) METS-IR, a novel score to evaluate insulin sensitivity, is predictive of visceral adiposity and incident type 2 diabetes. Eur J Endocrinol 178(5):533-544. https://doi.org/10.1530/EJE-17-0883 
21. Bochaliya RK, Sharma A, Saxena P, Ramchandani GD, Mathur G (2019) To evaluate the association of neck circumference with metabolic syndrome and cardiovascular risk factors. J Assoc Physicians India 67(3):60-62

22. Laohabut I, Udol K, Phisalprapa P, Srivanichakorn W, Chaisathaphol T, Washirasaksiri C et al (2020) Neck circumference as a predictor of metabolic syndrome: a cross-sectional study. Prim Care Diabetes 14(3):265-273. https://doi.org/10.1016/j.pcd. 2019.08.007

23. Mancia G, Fagard R, Narkiewicz K, Redon J, Zanchetti A, Böhm $\mathrm{M}$ et al (2013) $2013 \mathrm{ESH} / \mathrm{ESC}$ guidelines for the management of arterial hypertension: the task force for the management of arterial hypertension of the European Society of Hypertension (ESH) and of the European Society of Cardiology (ESC). Eur Heart J 34(28):2159-2219. https://doi.org/10.1093/eurheartj/eht151

24. Alberti KGMM, Eckel RH, Grundy SM, Zimmet PZ, Cleeman JI, Donato KA et al (2009) Harmonizing the metabolic syndrome: a joint interim statement of the International Diabetes Federation Task Force on Epidemiology and Prevention; National Heart, Lung, and Blood Institute; American Heart Association; World Heart Federation; International Atherosclerosis Society; and International Association for the Study of Obesity. Circulation 120(16):1640-1645. https://doi.org/10.1161/CIRCULATIO NAHA.109.192644

25. Lim J, Kim J, Koo SH, Kwon GC (2019) Comparison of triglyceride glucose index, and related parameters to predict insulin resistance in Korean adults: an analysis of the 2007-2010 Korean
National Health and Nutrition Examination Survey. PLoS ONE 14(3):e0212963. https://doi.org/10.1371/journal.pone.0212963

26. Yu X, Wang L, Zhang W, Ming J, Jia A, Xu S et al (2019) Fasting triglycerides and glucose index is more suitable for the identification of metabolically unhealthy individuals in the Chinese adult population: a nationwide study. J Diabetes Investig 10(4):1050 1058. https://doi.org/10.1111/jdi.12975

27. Yang G-R, Dye TD, Zand MS, Fogg TT, Yuan S-Y, Yang J-K et al (2019) Association between neck circumference and coronary heart disease: a meta-analysis. Asian Pac Isl Nurs J 4(1):34-46. https://doi.org/10.31372/20190401.1031

28. Caro P, Guerra X, Canals A, Weisstaub G, Sandaña C (2019) Is neck circumference an appropriate tool to predict cardiovascular risk in clinical practice? A cross-sectional study in Chilean population. BMJ Open 9(11):e028305. https://doi.org/10.1136/bmjop en-2018-028305

29. Mendes CG, Barbalho SM, Tofano RJ, Lopes G, Quesada KR, Detregiachi CRP et al (2021) Is neck circumference as reliable as waist circumference for determining metabolic syndrome? Metab Syndr Relat Disord 19(1):32-38. https://doi.org/10.1089/ met.2020.0083

Publisher's Note Springer Nature remains neutral with regard to jurisdictional claims in published maps and institutional affiliations. 